\title{
Can studies of harm be harmful?
}

\author{
Joel G. Ray MD MSc
}

$\infty$ See related research paper by Wen and colleagues, page 1263

I $\mathrm{n}$ this issue of $C M A J$, Wen and colleagues report that the use of folic acid antagonists was associated with a higher risk of placenta-mediated adverse outcomes of pregnancy. ${ }^{1}$ Had these medications appeared to be protective, we might be more reluctant to accept the association as causal or to label the findings as "ready for prime time." Rather, we would make the usual comment that a randomized controlled trial is needed to prove efficacy. Although rigorous randomized trials are required to demonstrate therapeutic efficacy, we usually have little choice but to resort to more biased observational study designs to investigate undesirable effects. As noted by Wen and colleagues, randomized trials of therapeutic harm are rarely ethical and are certainly not practical, especially during pregnancy.

The use of nonrandomized epidemiologic studies to evaluate harm is not a major problem. However, they too must be rigorous: they should accurately capture both the exposure and outcomes of interest, identify the correct temporal relations and account for major confounding variables. Such studies must also show estimates of risk that are clinically relevant rather than just statistically relevant. The study by Wen and colleagues should be included in academic discussions, but it should not be adopted by clinical practitioners or policy-makers. This study does not convincingly meet these requirements because of the 4 following reasons.

The first reason relates to the study exposure. Wen and colleagues postulated that the use of folic acid antagonists may be associated with placenta-mediated adverse outcomes of pregnancy, similar to those caused by folate deficiency. ${ }^{2}$ The authors examined the use of dihydrofolate-reductase inhibitors and other folate antagonists. Antibiotics containing trimethoprim and certain older generation anticonvulsants comprised $68 \%$ and $20 \%$ of all study medications, respectively. Therein lies $88 \%$ of the problem.

In the study by Wen and colleagues, a folic acid antagonist may have been dispensed up to 1 year before delivery of a single infant. This means that a woman may have taken the medication up to 3 months before conception and for a duration as short as 1-7 days. Moreover, $49 \%$ of all trimethoprimbased antibiotics were dispensed before conception. It is hard to believe that taking trimethoprim for just a few days well before conception can have a lasting effect on folate metabolism or the vascular integrity of the nonexistent syncytiotrophoblasts or placental spiral arteries. Restriction of the analysis to drug exposure during the first and second trimester did not appreciably alter the risk estimates.

Most participants who were prescribed more than

\section{Key points}

- Folate antagonists may not have been prescribed close enough to the time of pregnancy or for long enough to affect placental function.

- Folate antagonists were not prescribed randomly; thus, the results may be biased.

- Trimethoprim and antiepileptic medications should continue to be used before or during pregnancy because they prevent maternal illness.

20 tablets were probably either immunocompromised (e.g., HIV-positive patients who required trimethoprimsulphamethoxazole for pneumocystis jiroveci prophylaxis) or had epilepsy and required antiseizure medication. This is important because the authors describe a dose-response relation between the number of pills dispensed and the risk of an adverse outcome. A dose-response relation may heighten the plausibility of a causal association; however, in the study by Wen and colleagues, a higher number of pills dispensed (and therefore a longer duration of therapy) probably reflected an underlying chronic condition likely to negatively affect maternal and fetal well-being.

The second reason that this study does not persuade us to change practice relates to the study outcomes. The reported rates of preeclampsia, placental abruption and other outcomes are consistent with other high-quality epidemiologic studies, which suggests that the outcomes were properly ascertained. However, the authors did not report how many women and their infants were counted more than once because of consecutive pregnancies or the occurrence of multiple outcomes. The inclusion of women at high risk of an outcome occurring more than once could inflate the risk estimates, because placenta-mediated conditions often reoccur. Additionally, the number of offspring with a congenital anomaly that is sensitive to folic acid was not reported. This is relevant, given that infants with birth defects are at higher risk of growth restriction. ${ }^{3}$

The third reason relates to reported effect sizes. The rate of use of folic acid antagonists among all women who delivered during the study period was not reported. Thus, we cannot be confident about the true prevalence of the exposure. However, Wen and colleagues previously observed a rate of $8.4 \%$

Joel Ray is with the Departments of Medicine, Obstetrics and Gynecology and Health Policy Management and Evaluation, St. Michael's Hospital and the Institute for Clinical Evaluative Sciences, University of Toronto, Toronto, Ont. 
for use of dihydrofolate-reductase inhibitors and $1.1 \%$ for other folic acid antagonists among women of reproductive age in the same area. ${ }^{4}$ Using the combined figure of $9.5 \%$ for exposure to folate-acid antagonists and the current findings of an adjusted odds ratio of 1.77, the population attributable risk for severe preeclampsia from exposure to these drugs is $6.8 \%$ (the overall risk of severe preeclampsia that might be attributed to use of folic acid antagonists). ${ }^{5}$ Based on an odds ratio of 1.35 for fetal death, the calculated population attributable risk percent is $4.3 \%$.

The final issue is confounding, which the authors have acknowledged. Although Wen and colleagues adjusted for a limited number of potential confounding variables (e.g., maternal age, parity), they only presented the adjusted odds ratios. Without knowing the crude odds ratios, we cannot determine the degree to which these variables influenced the relation between use of folate antagonists and adverse outcome during pregnancy and, thus, the degree to which they behaved as confounders.

Importantly, some potentially critical confounding variables, including use of folic acid supplements or the presence of maternal diabetes mellitus and hypertension, were not ascertained by Wen and colleagues. Receipt of an antibiotic containing trimethoprim may have been a proxy for the presence of maternal diabetes mellitus, because asymptomatic bacteriuria is more prevalent among women with diabetes mellitus. ${ }^{6}$ This is important, because obese women and those with diabetes are at higher risk than nonobese women for placenta-mediated adverse outcomes of pregnancy. ${ }^{7.8}$ Similarly, the receipt of the combination antihypertensive drug triamterene-hydrochlorthiazide, which comprised about $11 \%$ of all dispensed folate antagonists, could have been a proxy for either chronic hypertension (29\% received the drug before conception, and $17 \%$ and $14 \%$ received the drug in the first and second trimesters, respectively) or pregnancy-induced hypertension $(40 \%$ received the drug during the third trimester). The near tripling in the rate of use of triamterenehydrochlorthiazide between the second and third trimesters suggests that women were receiving treatment for pregnancyinduced hypertension, yet preeclampsia was a study outcome. Moreover, all forms of hypertension during pregnancy are associated with a substantially higher risk of placental abruption, fetal growth restriction and fetal death, especially when an antihypertensive agent is required. ${ }^{9}$

How can we untangle whether it is a maternal attribute or the folic acid antagonist that is associated with placentamediated disease? One way would be to also include use of other prescription drugs used to treat these conditions but that do not interfere with folate metabolism. This would help to reduce the major threat to validity introduced by confounding by indication in observational studies of drug therapy.

Although there is a small possibility that the use of trimethoprim-based antibiotics before or during pregnancy slightly increases the risk of placenta-mediated disease, these agents should continue to be prescribed to women planning a pregnancy or who are currently pregnant. The treatment of asymptomatic bacteriuria prevents maternal pyelonephritis and some adverse perinatal outcomes. ${ }^{10}$ Trimethoprimsulfamethoxazole remains a first-line agent for the treatment of urinary tract infections among women allergic to penicillin. For chronic conditions such as epilepsy, for which long-term use of antiseizure medication is warranted, the use of folic acid supplements before conception along with a single agent at its lowest therapeutic dose to properly control seizure activity is strongly recommended. ${ }^{11}$

Competing interests: None declared.

\section{REFERENCES}

1. Wen SW, Zhou J, Yang Q, et al. Maternal exposure to folic acid antagonists and placenta-mediated adverse pregnancy outcomes. CMAJ 2009;179:1263-8.

2. Mignini LE, Latthe PM, Villar J, et al. Mapping the theories of preeclampsia: the role of homocysteine. Obstet Gynecol 2005;105:411-25.

3. Paulozzi LJ. The relation between small size for gestational age and the sex ratio of children with birth defects. Teratology 2001;63:52-6.

4. Wen SW, Walker M. Risk of fetal exposure to folic acid antagonists. J Obstet Gynaecol Can 2004;26:475-80.

5. Rockhill B, Newman B, Weinberg C. Use and misuse of population attributable fractions. Am J Public Health 1998;88:15-9.

6. Geerlings SE, Stolk RP, Camps MJ, et al. Asymptomatic bacteriuria may be considered a complication in women with diabetes. Diabetes Mellitus Women Asymptomatic Bacteriuria Utrecht Study Group. Diabetes Care 2000;23:744-9.

7. Odegard RA, Vatten LJ, Nilsen ST, et al. Risk factors and clinical manifestations of pre-eclampsia. BJOG 2000;107:1410-6.

8. Ray JG, Vermeulen MJ, Shapiro JL, et al. Maternal and neonatal outcomes in pregestational and gestational diabetes mellitus, and the influence of maternal obesity and weight gain: the DEPOSIT study. Diabetes Endocrine Pregnancy Outcome Study in Toronto. QJM 2001;94:347-56.

9. Ray JG, Vermeulen MJ, Burrows EA, et al. Use of antihypertensive medications in pregnancy and the risk of adverse perinatal outcomes: McMaster Outcome Study of Hypertension In Pregnancy 2 (MOS HIP 2). BMC Pregnancy Childbirth 2001;1:6.

10. Smaill F, Vazquez JC. Antibiotics for asymptomatic bacteriuria in pregnancy. Cochrane Database Syst Rev 2007;(2):CD000490.

11. Meador KJ, Pennell PB, Harder Cl, et al; HOPE Work Group. Pregnancy registries in epilepsy. A consensus statement on health outcomes. Neurology 2008:71;110917.

Correspondence to: Dr. Joel G. Ray, Department of Medicine, St. Michael's Hospital, 30 Bond St., Toronto ON M5B 1W8; fax 416 864-5485; rayj@smh.toronto.on.ca 